\title{
Coordination geometry preference regulates structure and dynamics of metallo-supramolecular polymer networks
}

\author{
Mostafa Ahmadi, Sebastian Seiffert ${ }^{*}$ \\ Department of Chemistry, Johannes Gutenberg-Universität Mainz, Duesbergweg 10-14, D-55128 Mainz, \\ Germany \\ *Correspondence to Sebastian Seiffert (sebastian.seiffert@uni-mainz.de)
}

\section{- Supporting Information -}

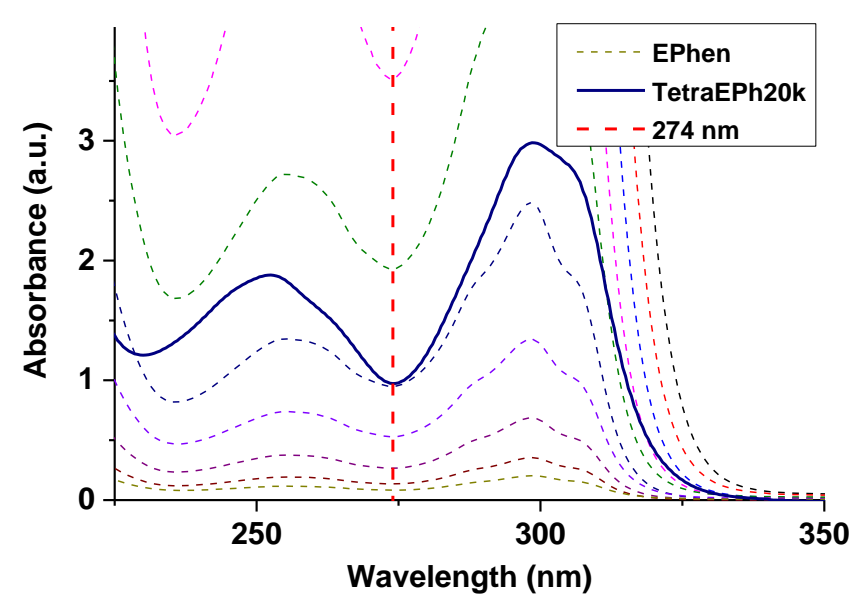

Figure S1. UV-vis spectra of the small-molecule EPhen ligand in methanol at different concentrations (dashed lines) and that of the tetraEPh20k precursor. The absorption band at $274 \mathrm{~nm}$ is used to build the calibration curve and estimate the functionalization degree. 

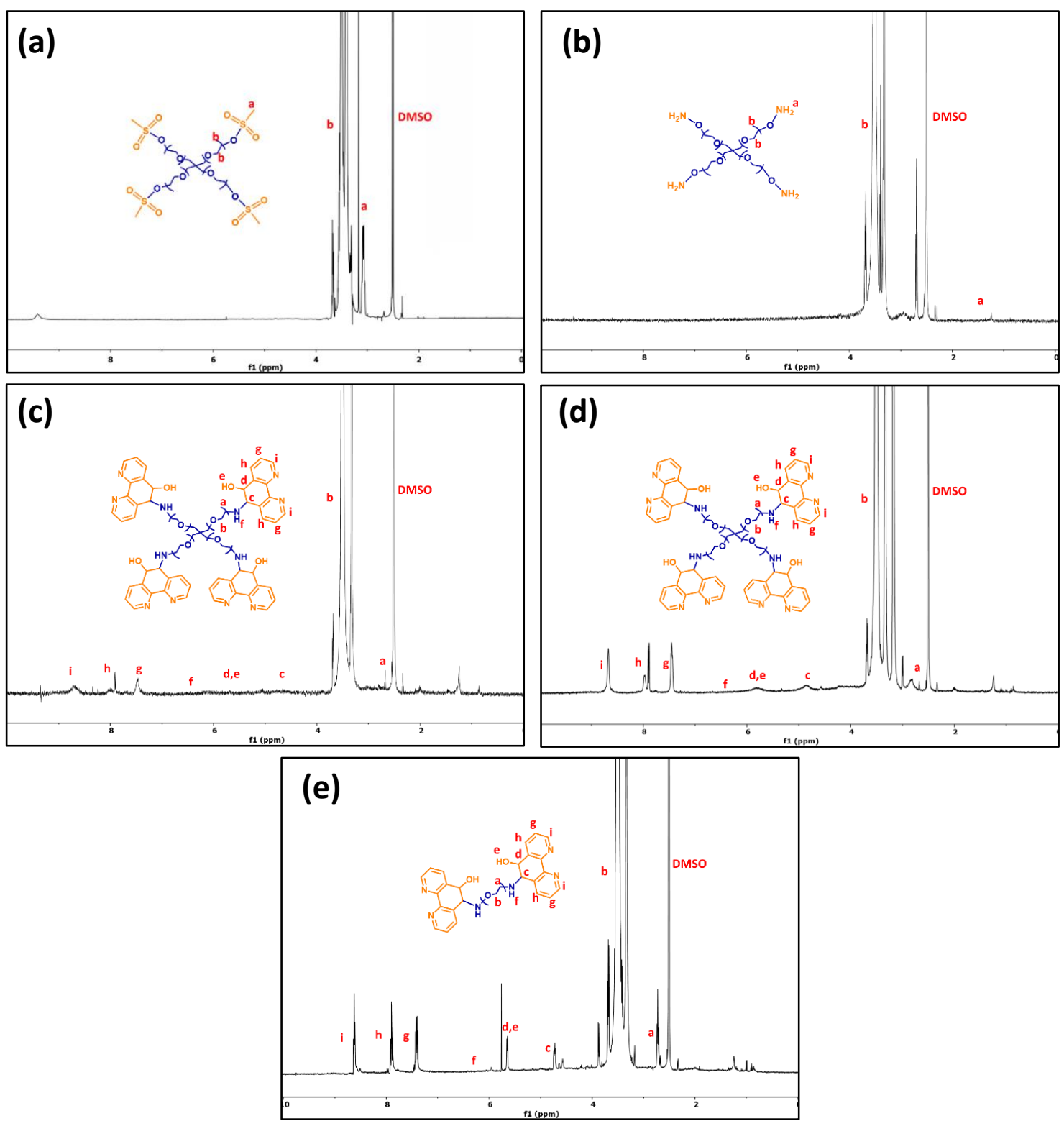

Figure S2. ${ }^{1} \mathrm{H}$ NMR spectra (400 MHz, DMSO- $d_{6}$ ) of (a) mesyl functionalized tetraPEG20k, (b) amine functionalized tetraPEG20k, (c) tetraEPh20k, (d) tetraEPh10k, and (e) bisEPh10k. 

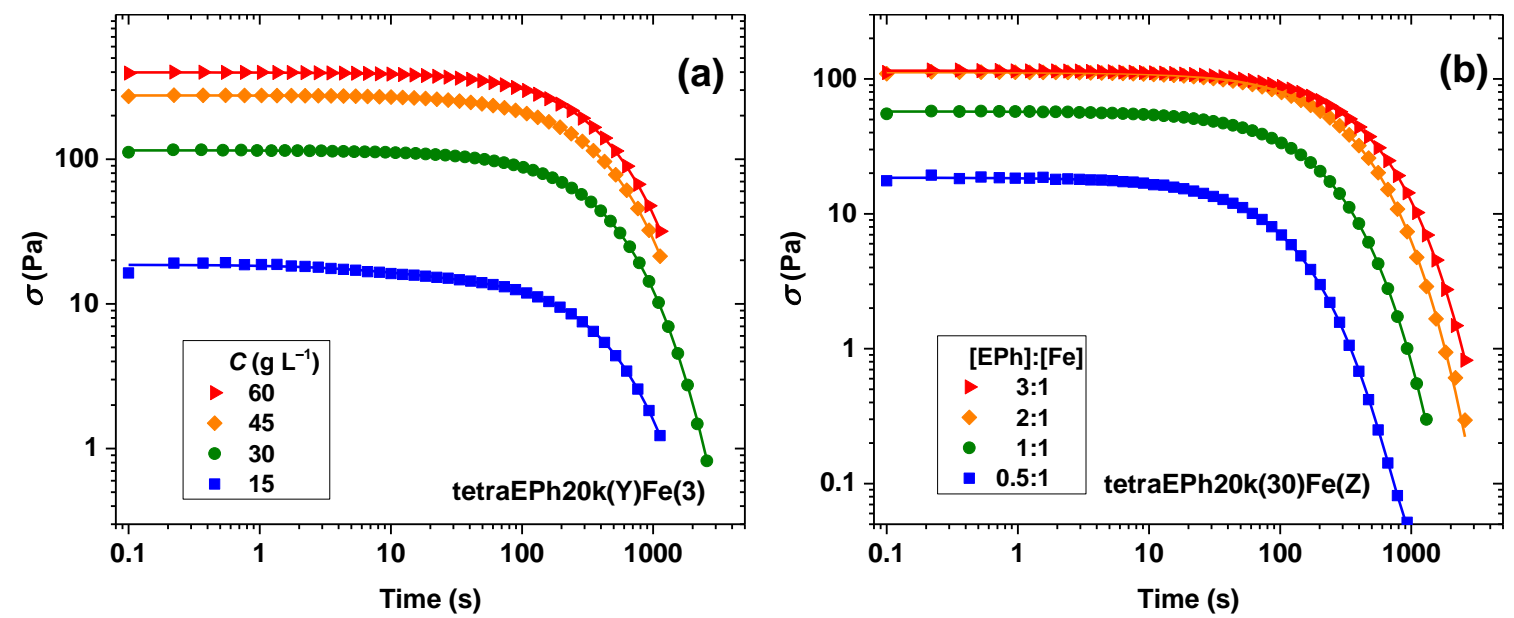

Figure S3. Shear stress of transient networks obtained by complexing tetraEPh20k with $\mathrm{Fe}^{2+}:(\mathrm{a})$ effect of the polymer concentration at [EPh]:[Fe] $=3$, and (b) effect of the stoichiometric ratio at $\varphi=30 \mathrm{~g} \mathrm{~L}^{-1}$. Solid lines are fitted curves obtained according to eqn (2) and (3).
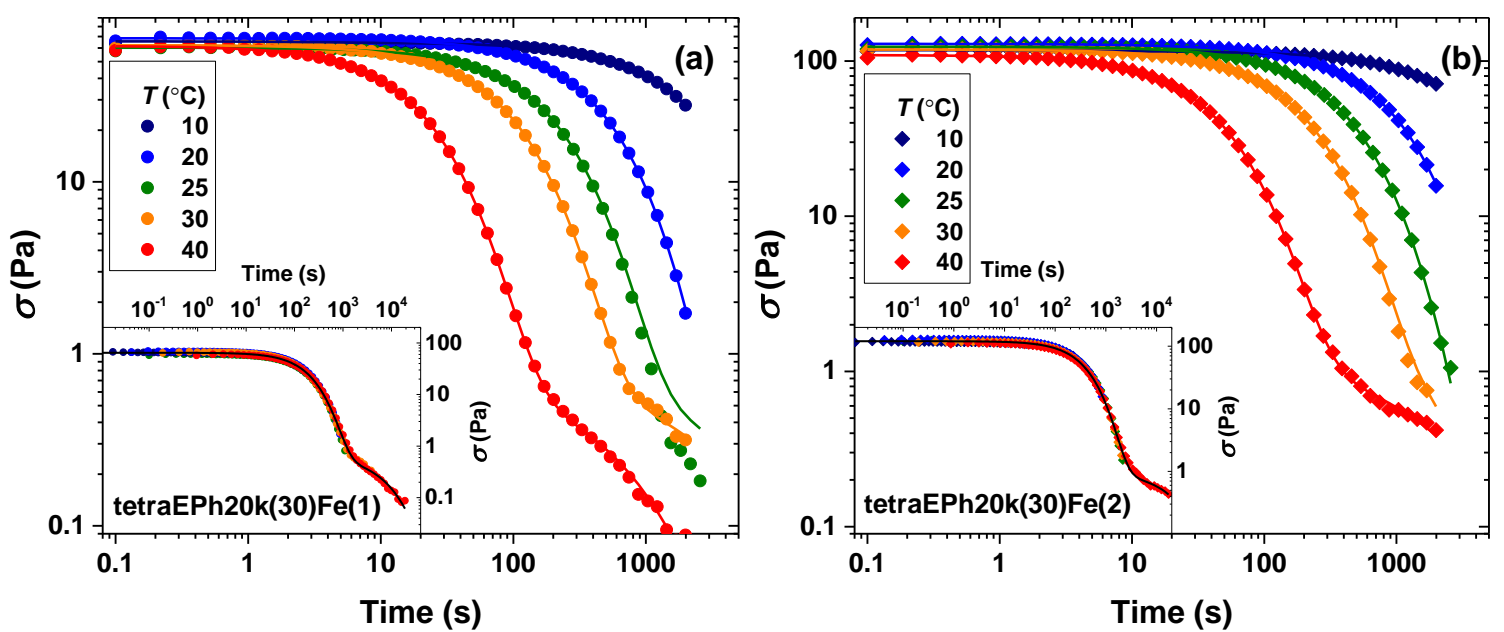

Figure S4. Shear stress of transient networks obtained by tetraEPh20k at $\varphi=30 \mathrm{~g} \mathrm{~L}^{-1}$ and Fe stoichiometric ratios: (a) $[\mathrm{EPh}]:[\mathrm{Fe}]=1$, and $(\mathrm{b})[\mathrm{EPh}]:[\mathrm{Fe}]=\mathbf{2}$. Solid lines are fitted curves obtained according to eqn (2) and (3) (main plot). Master-curves obtained by the horizontal shift of data acquired at different temperatures to $25^{\circ} \mathrm{C}$ (inset plot). 



Figure S5. Dynamic storage (filled symbol and solid lines) and loss (open symbols and dashed lines) moduli of transient networks built with tetraEPh10k: effect of the polymer concentration on rheological behavior of networks formed by (a) $\mathrm{Fe}^{2+}$, (b) $\mathrm{Co}^{2+}$ at $[\mathrm{EPh}]:[\mathrm{M}]=3$, and effect of the stoichiometric ratio on rheological behavior of networks formed by (c) $\mathrm{Fe}^{2+}$, and (d) $\mathrm{Co}^{2+}$ at $\varphi=60 \mathrm{~g} \mathrm{~L}^{-1}$.
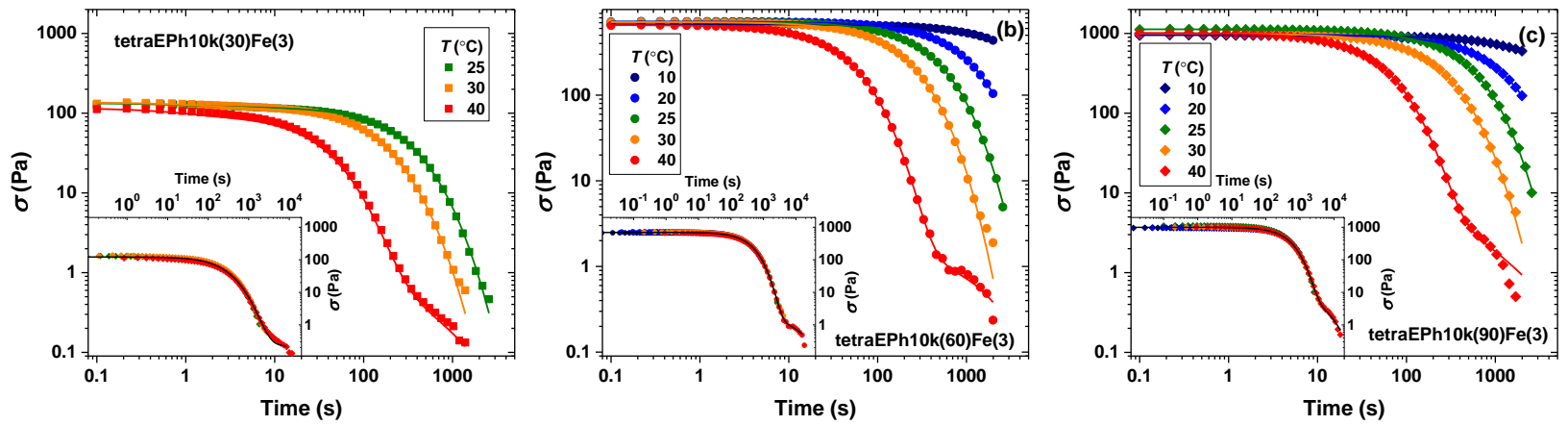

Figure S6. Shear stress of transient networks obtained by complexing tetraEPh10k at $[\mathrm{EPh}]:[\mathrm{Fe}]=3$ at different concentrations: (a) $\varphi=30 \mathrm{~g} \mathrm{~L}^{-1}$, (b) $\varphi=60 \mathrm{~g} \mathrm{~L}^{-1}$, and (c) $\varphi=90 \mathrm{~g} \mathrm{~L}^{-1}$. Solid lines are fitted curves obtained according 
to eqn (2) and (3) (main plot). Master-curves obtained by the horizontal shift of data acquired at different temperatures to $25^{\circ} \mathrm{C}$ (inset plot).


Figure S7. Visual appearance of samples made by complexation of tetraEPh20k at $\varphi=30 \mathrm{~g} \mathrm{~L}^{-1}$ as a function of stoichiometric ratio, as noted on the sample labels, for (upper) $\mathrm{Fe}^{2+}$, which forms a gel at [EPh]:[Fe] values below 3 , and (lower) $\mathrm{Co}^{2+}$, which forms a gel at [EPh]:[Co] values above 2.
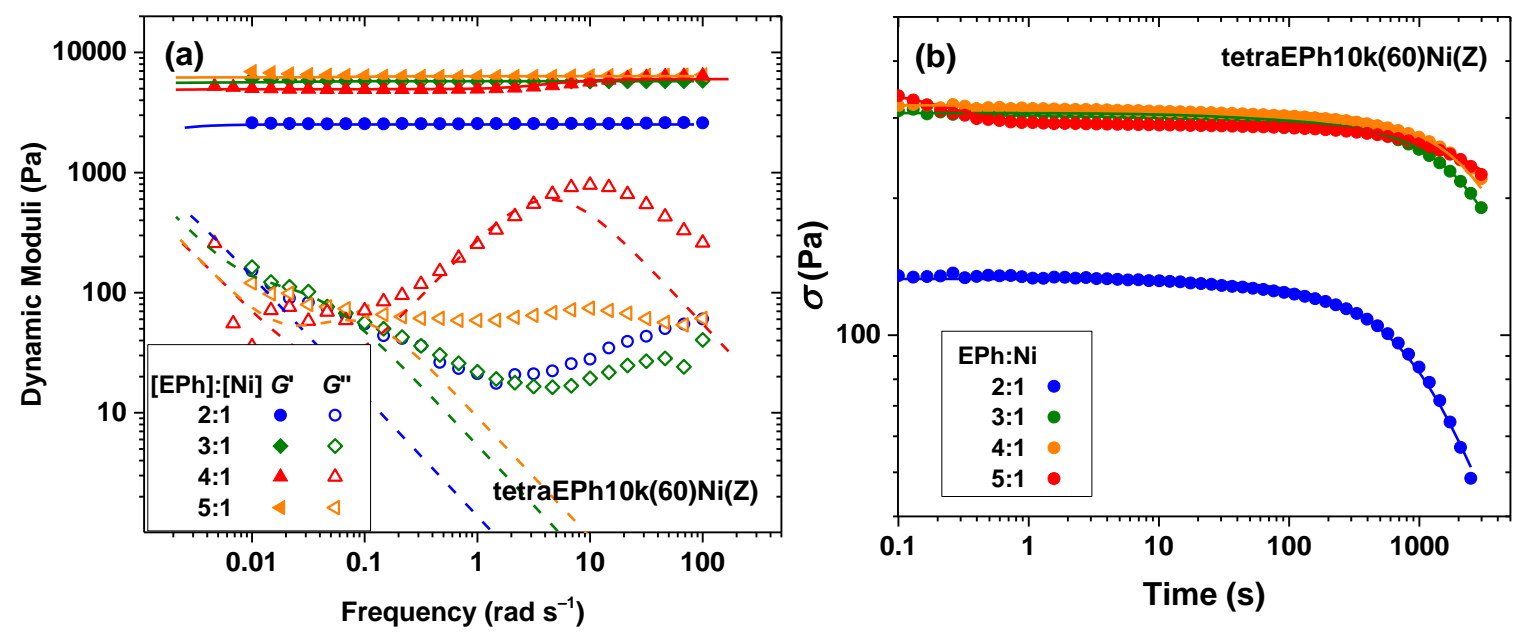

Figure S8. (a) Dynamic storage (filled symbol and solid lines) and loss (open symbols and dashed lines) moduli of transient networks built by tetraEPh10k at $\varphi=60 \mathrm{~g} \mathrm{~L}^{-1}$ with $\mathrm{Ni}^{2+}$ at different stoichiometric ratios. Solid lines are obtained according to eqn (4) and (5). (b) the corresponding Shear stresses achieved from the step-strain measurement. 

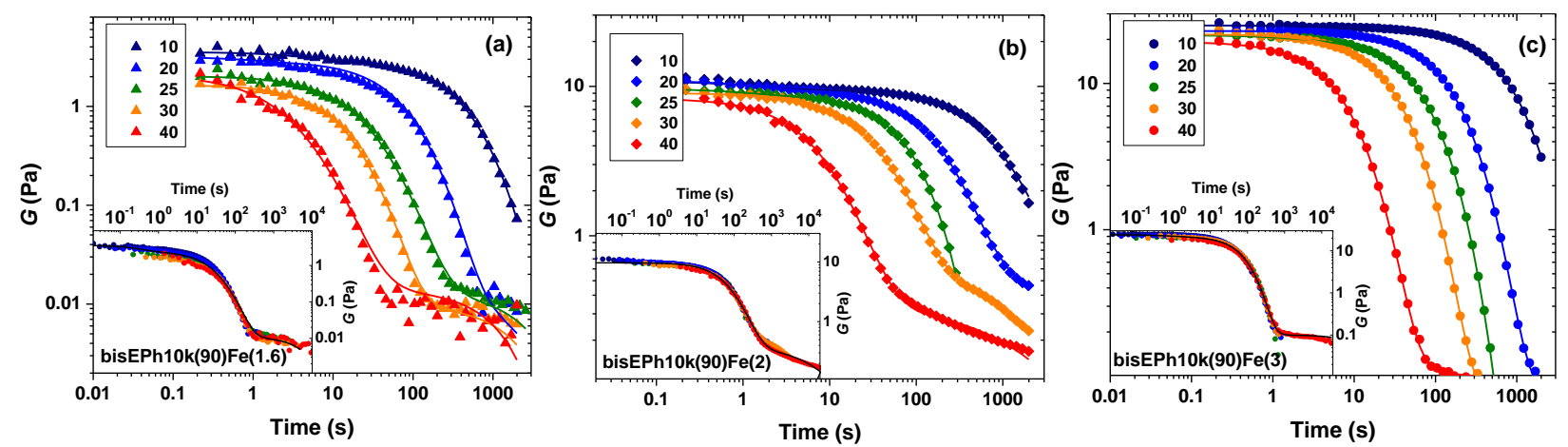

Figure 59. Shear stress of transient networks formed by bisEPh10k at $\varphi=90 \mathrm{~g} \mathrm{~L}^{-1}$ with $\mathrm{Fe}^{2+}$ at different stoichiometric ratios: (a) $[E P h]:[F e]=1.6,(b)[E P h]:[F e]=2$, and (c) $[E P h]:[F e]=3$. Solid lines are fitted curves obtained according to eqn (2) and (3) (main plot). Master-curves obtained by the horizontal shift of data acquired at different temperatures to $25^{\circ} \mathrm{C}$ (inset plot).
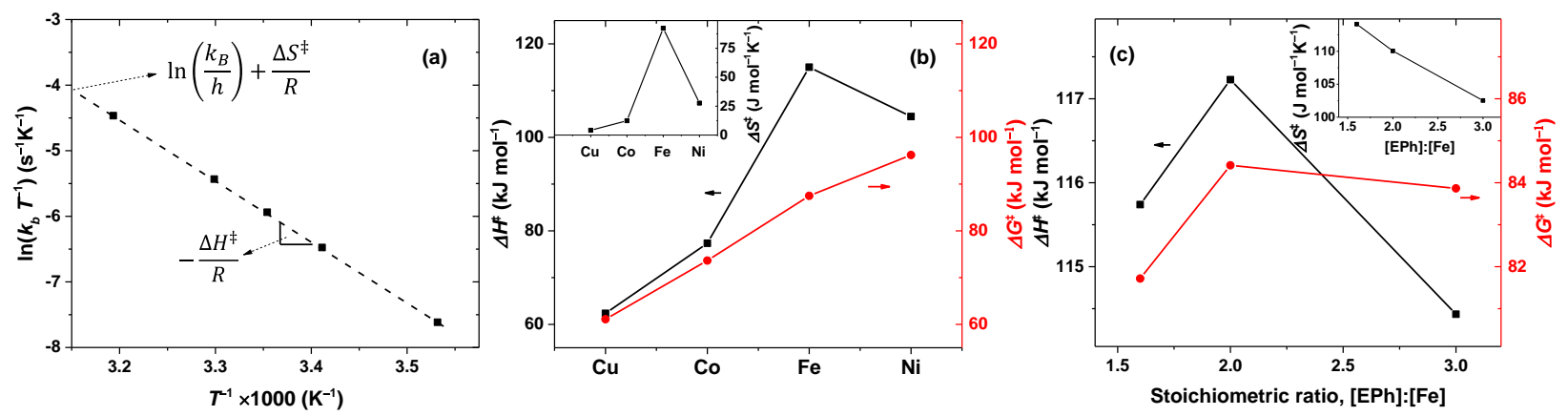

Figure S10. (a) Demonstration of the utility of the Eyring's equation to decouple the enthalpy and entropy of activation of dissociation reaction for tetraEPh20k(30)Co(3) sample. (b) Gibbs free energy of activation and enthalpy of activation of tetraEPh20k(30)M(3) networks for different metal ions (main plot) and the corresponding entropy of activation (inset plot). (c) Gibbs free energy of activation and enthalpy of activation of bisEPh10k(90)Fe networks for different stoichiometric ratios (main plot) and the corresponding entropy of activation (inset plot). 
Table S1. Calculated equilibrium constants of bis- and tris-complexes according to different fitting scenarios shown in Figure 8d.

\begin{tabular}{|l|c|c|c|}
\hline Considered complexes & $\log \left(K_{1}\right)$ & $\log \left(K_{2}\right)$ & $\log \left(K_{3}\right)$ \\
\hline Bis+Tris & \multicolumn{3}{|c|}{ Co } \\
\hline Bis & 2.90 & 1.60 & 5.78 \\
\hline Tris & 3.50 & 3.86 & 0.00 \\
\hline & 2.35 & 4.05 & 3.66 \\
\hline Bis+Tris & \multicolumn{3}{|c|}{ Fe } \\
\hline Bis & 7.29 & 6.53 & 7.18 \\
\hline Tris & 5.10 & 4.25 & 0.00 \\
\hline & 6.78 & 4.59 & 8.98 \\
\hline Bis+Tris & & $\mathbf{N i}$ \\
\hline Bis & 3.12 & 4.20 & 4.66 \\
\hline Tris & 3.79 & 4.15 & 0.00 \\
\hline
\end{tabular}



Figure S11. Optimized structures of (a) Co-bis(Phen) , (b) Fe-bis(Phen), (c) Ni-bis(Phen), (d) Co-tris(Phen), (e) Fe-tris(Phen), (f) Ni-tris(Phen). 
Table S2. Bond length between nitrogen atoms of ligands and coordinated metal ions. Three bond lengths of the tris-complex designate that of the symmetric ligand and the short and long bond lengths of the asymmetric ligand.

\begin{tabular}{|l|l|l|l|}
\hline Bond length & Co & Fe & Ni \\
\hline Mono & 1.8958 & 1.9168 & 1.8676 \\
\hline Bis & 1.9795 & 1.9850 & 1.9448 \\
\hline Tris (symmetrically coordinated ligand) & 2.0080 & 2.0195 & 1.9650 \\
\hline Tris (asymmetrically coordinated ligand) & 2.0113 & 2.0247 & 1.9567 \\
\hline Tris (asymmetrically coordinated ligand) & 2.0252 & 2.0275 & 2.5895 \\
\hline
\end{tabular}

Table S3. Sum of electronic and thermal Free Energies of the optimized structures ( $\left.\mathrm{kcal} \mathrm{mol}^{-1}\right)$.

\begin{tabular}{|c|c|c|c|}
\hline Complex & Co & Fe & Ni \\
\hline Mono & -1953.62 & -1834.52 & -2079.15 \\
\hline Bis & -2525.26 & -2406.14 & -2650.8 \\
\hline Tris & -3097.24 & -2978.21 & -3222.75 \\
\hline
\end{tabular}
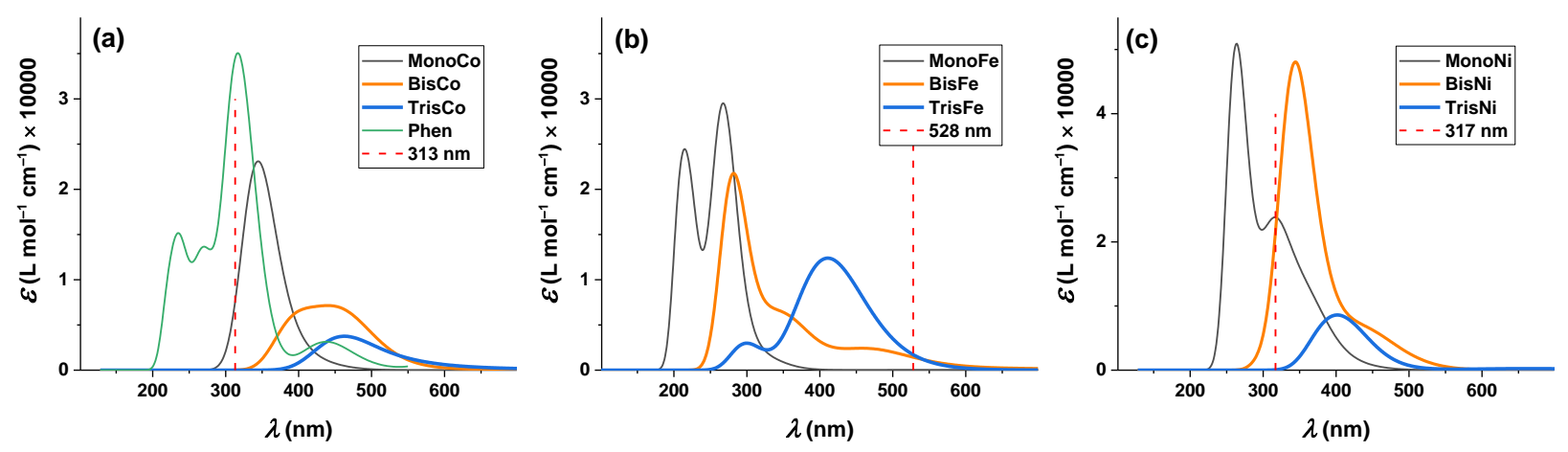

Figure S12. Molar extinction coefficients of metal-ligand complexes with different coordination geometries made by (a) $\mathrm{Co}^{2+}$, (b) $\mathrm{Fe}^{2+}$, and (c) $\mathrm{Ni}^{2+}$ metal ions. The specific absorption bands, which were used to follow the stoichiometric ratio dependence of complexation in titration experiments are indicated by dashed lines. 